\title{
Selected Papers from TopSafe 2008: Safety at Nuclear Installations
}

\author{
Michel Giot, ${ }^{1}$ Nikola Čavlina, ${ }^{2}$ Dubravko Pevec, ${ }^{2}$ and Alessandro Petruzzi ${ }^{3}$ \\ ${ }^{1}$ Louvain School of Engineering, Université Catholique de Louvain, 1348 Louvain-la-Neuve, Belgium \\ ${ }^{2}$ Faculty of Electrical Engineering and Computing, University of Zagreb, 1000 Zagreb, Croatia \\ ${ }^{3}$ Department of Mechanical, Nuclear and Production Engineering, University of Pisa, 56126 Pisa, Italy
}

Correspondence should be addressed to Michel Giot, michel.giot@uclouvain.be

Received 12 April 2010; Accepted 12 April 2010

Copyright ( $) 2010$ Michel Giot et al. This is an open access article distributed under the Creative Commons Attribution License, which permits unrestricted use, distribution, and reproduction in any medium, provided the original work is properly cited.

At the end of 2008, a conference on the safety of nuclear installations was successfully organized in Dubrovnik by the European Nuclear Society (ENS). A selection of the papers of this TOPSAFE Conference is proposed in this special issue after the usual long review process. We thank the authors who submitted a revised version of their paper and the distinguished scientists who accepted to review them carefully.

The selected papers of the TOPSAFE Conference presented here are grouped around six themes, all very relevant to safety. A first series of three papers deals with the role of nuclear safety codes in general. Presenting several examples of applications, the paper by Thomas Höhne et al. of the Research Centre of Dresden-Rossendorf emphasizes the role of CFD as a tool in the detailed analysis of safety issues of Pressurized Water Reactors, like coolant mixing and stratification, debris transport phenomena, and subcooled boiling in a fuel rod bundle. The quality requirements of the models contained in the CFD codes are outlined. Among the system codes, the advanced capabilities of RELAP/ SCDAPSIM are presented in the paper by Chris Allison and Judith K. Hohorst of Innovative Systems Software, LLC. Besides improvements of the models included in the code, improvements in coding and numerics enable the code to be run on complex multidimensional problems faster than real time on inexpensive personal computers and to be used to support training activities. Finally, the paper by Eugenijus Ušpuras of the Lithuanian Energy Institute illustrates the impact of a set of deterministic and probabilistic studies on the decisions regarding the Ignalina NPP safety improvement program.
Containment integrity in a severe accident is the subject of the next two papers proposed by IRSN (Institut de Radioprotection et Sûreté Nucléaire). Ahmed Bentaib et al. present a methodology and its application to Level 2 Probabilistic Safety Assessment to evaluate the impact of the passive autocatalytic recombiners on the hydrogen risk. A set of 35 scenarios have been examined and, subject to further experimental verification, it is concluded that the use of recombiners significantly reduces but does not totally eliminate the risk of flame acceleration and transition to detonation. The other paper on containment integrity, by Renaud Meignen and Tanguy Janin, deals with direct containment heating that could occur after the eventual failure of the reactor vessel. This process is related to dynamical, thermal, and chemical phenomena associated with the possible fine fragmentation and dispersal of the corium melt out of the vessel pit. It may threaten the integrity of the containment by pressurization of its atmosphere. Here the MC3D code has been used. Further developments will require a model for the combustion of hydrogen.

Other aspects of severe accidents are studied in the next series of three papers. A comparison between two codes, RELAP5/SCDAPSIM and MAAP4, has been performed by Siniša Šadek et al. in the framework of cooperation between the University of Zagreb, ENCONET Company and NPP Krško. The case study postulates a blackout of the NPP with on-site unavailable supply and with a leakage from reactor coolant pump seals, leading to core degradation. It is shown that both phenomenological and mechanistic models in severe accident codes (MAAP4 and RELAP5/SCDAPSIM) have their own benefits and drawbacks. Their usage can pose 
challenges to the user, in first case due to need for model parameters selection and in second case due to more detailed input preparation.

A step for the uncertainty analysis of large-break LOCAs is presented in the paper of Francesc Reventos et al. showing the results of the BEMUSE (Best Estimate Methods plus Uncertainty and Sensitivity Evaluation) programme. This programme involves 13 participants using different codes or different versions of codes.

Nowadays the increased industrial competition and constraints result in more aggressive conditions for the fuel (higher burnup, higher power, load follow, ...) and create incentive conditions for the development of advanced fuel designs with improved performance (new fuel types with additives, cladding material with better resistance to corrosion, ...). These developments involve the need for new investigations of irradiated fuel behavior under reference accidents in order to check the adequacy of the current criteria, evaluate the safety margins, provide new technical bases for modelling, and allow an evolution of these criteria. The paper by François Barré et al. discusses this issue and presents the IRSN strategy.

The next three papers deal with the safety of VVERs. The paper by Ivan Spasov et al. (INRNE-Sofia and IRSN) presents validation results for multichannel vessel thermal-hydraulic models in CATHARE used in coupled 3D neutronic/thermal hydraulic calculations. The test cases are from the OECD VVER-1000, coolant transient benchmark (V1000CT) and include asymmetric vessel flow transients and main steam line break (MSLB) transients. Another paper dealing with the qualification of coupled codes for reactor safety evaluations is proposed by Victor Sánchez et al. (FZK). In the frame of the VVER-1000, coolant transient benchmark RELAP5/PARCS has been extensively assessed and multidimensional thermal hydraulic phenomena as well as core physics were examined. Plant data were used to qualify the 3D models of TRACE and RELAP5/CFX, which were coupled for this purpose.

Finally, based on cooperation between IRSN and the University of Lappeenranta, the paper by Luben Sobitonov et al. summarizes the analysis results of three PACTEL experiments, carried out with the thermal-hydraulic system computer CATHARE 2 code. The three LOCA experiments, conducted on the Finnish test facility PACTEL (VVER-440 model), represent $7.4 \%$ cold leg breaks with combination of secondary bleed and primary bleed and feed and different actuation modes of the passive safety injection.

Two papers are devoted to the simulation of passive emergency heat removal systems. The paper by Lorenzo Santini et al. describes a new experimental facility built and operated at SIET laboratory. in Piacenza, while Davide Papini and Antonio Cammi report on their modeling of heat transfer phenomena for vertical or horizontal configurations of inpool condensers. A set of correlations are compared with data obtained with the PERSEO facility at SIET laboratory.

The last paper deals with RELAP5/MOD3.3 best estimate for human reliability. Proposed by Andrej Prošek and Borut Mavko of the Jožef Stefan Institute, this paper estimates the time windows for successful operator action in scenarios where the human actions are supplement to safety system actuations. The times needed for performing operator actions were determined based on simulator experience.

The editors are convinced that the broad spectrum of safety aspects dealt with in this special issue will be of interest to many readers of the journal.

Michel Giot Nikola Čavlina Dubravko Pevec Alessandro Petruzzi 

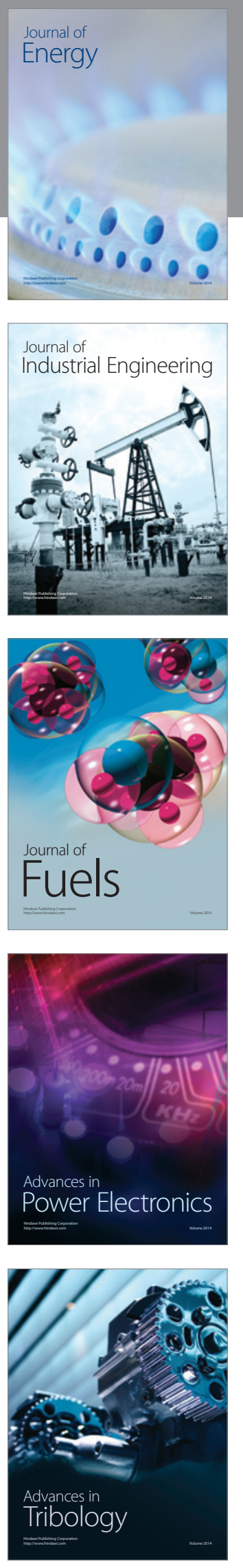
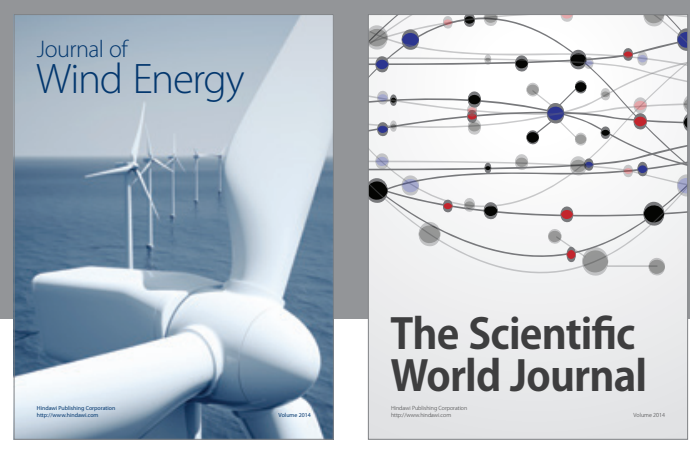

The Scientific World Journal

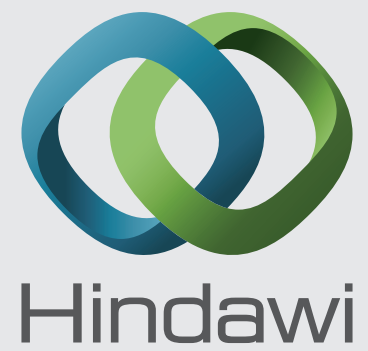

Submit your manuscripts at http://www.hindawi.com
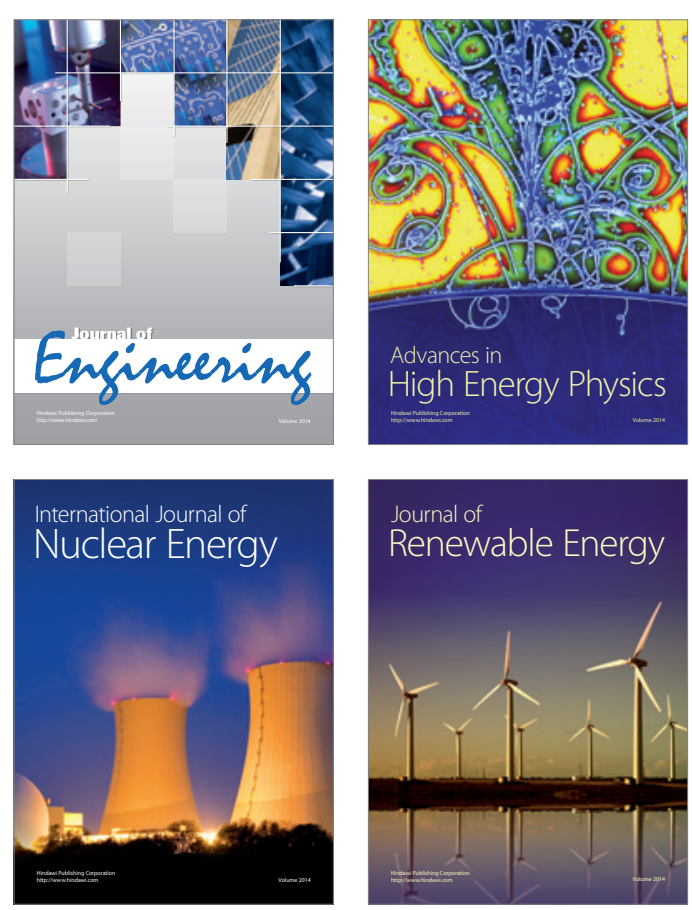

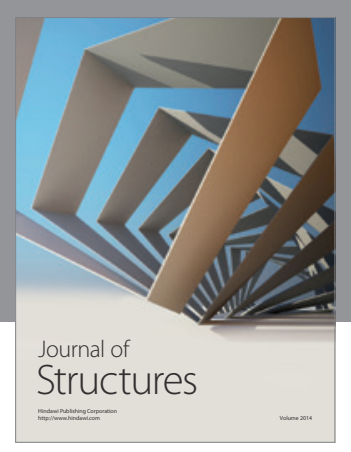

Rotating
Mechinery
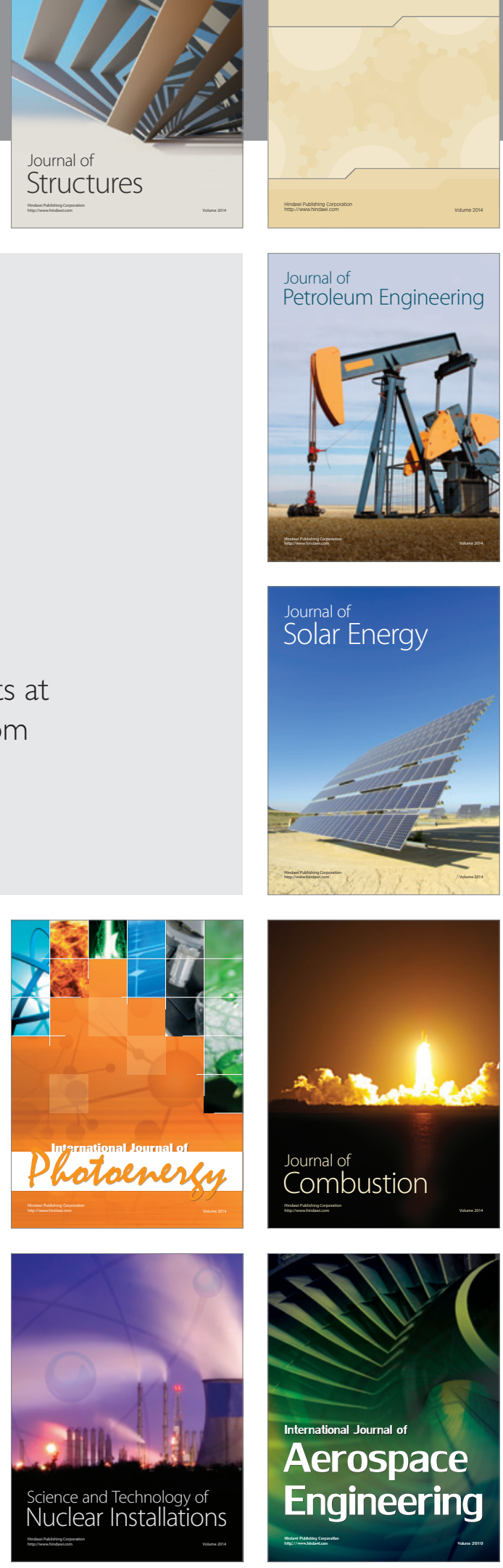